\title{
Effects of Full Order Geopotential Hessian on Precision Orbit Determination of Geodetic Satellites
}

\author{
John G. Warner * and Krysta Lemm* \\ US Naval Research Laboratory, Washington DC, 20375, United States
}

\begin{abstract}
Successful satellite operations often depend on the ability to precisely determine the satellite's position throughout it's operational lifetime. Often, simplified models are used to reduce the computational complexity when producing an orbit determination solution. One example is the use of lower order gravitational terms when computing the state transition matrix. The US Naval Research Laboratory's Orbit Covariance Estimation and Analysis software is used to characterize the affects of using the full order geopotential terms when calculating the state transition matrix. Orbit solutions are calculated for several geodetic satellite using satellite laser ranging data. The RMS of the error residual and number of iterations needed for convergence are used as metric to evaluate the difference of using the full order geopotential to calculate the state transition matrix. It is found that typically the RMS error is the same when the higher order gravitational terms are used; however, the number of iterations needed for convergence are often lower. Additionally, the difference in the orbit solution between both methods is examined. Surprisingly, these orbit solutions may differ by up to several meters. This indicates that the higher order gravitational terms should always be considered when calculating the state transition matrix. Because fewer iterations are often needed, this can result in an overall decrease in computational time required.
\end{abstract}

\section{Nomenclature}

x Satellite state vector

$\Re^{n}$ n-dimensional real space

$\mathbf{\Phi}$ Satellite state transition matrix

I State covariance matrix

A time varying dynamics matrix

$V$ the geopotential

$\Gamma$ the Hessian of the geopotential

$J_{2}$ the Earth's second zonal spherical harmonic gravity term

\section{Introduction}

Successful satellite operations are often dependent on the ability to precisely determine the satellite's position throughout the operational lifetime. Often, simplified models are used to reduce the computational complexity without sacrificing fidelity of the precision orbit determination solution. One such simplification is the use of only the $J_{2}$ order gravitational terms when computing the state transition matrix. Using the Orbit Covariance Estimation and ANalysis (OCEAN) precision orbit determination software, the affects of using the full order geopotential terms when calculating the state transition matrix is evaluated.

Precision orbit solutions are calculated for the geodetic satellites LAGEOS-1, LAGEOS-2, STELLA and STARLETTE using satellite laser ranging data. The orbit of these satellites is well suited for orbit determination studies since they have simple shapes and are passive in nature. By design, their orbits are dominated by gravitational forces. So, error in an orbit solution can be attributed to mis-modeling forces rather than to mis-modeling satellite attitude or maneuvers.

${ }^{*}$ Aerospace Engineer, Mission Development Branch, Washington, DC, AIAA Member 


\section{Report Documentation Page}

Public reporting burden for the collection of information is estimated to average 1 hour per response, including the time for reviewing instructions, searching existing data sources, gathering and maintaining the data needed, and completing and reviewing the collection of information. Send comments regarding this burden estimate or any other aspect of this collection of information, including suggestions for reducing this burden, to Washington Headquarters Services, Directorate for Information Operations and Reports, 1215 Jefferson Davis Highway, Suite 1204, Arlington VA 22202-4302. Respondents should be aware that notwithstanding any other provision of law, no person shall be subject to a penalty for failing to comply with a collection of information if it does not display a currently valid OMB control number.

\begin{tabular}{|c|c|c|}
\hline $\begin{array}{l}\text { 1. REPORT DATE } \\
\mathbf{0 1} \text { AUG } \mathbf{2 0 1 4}\end{array}$ & $\begin{array}{l}\text { 2. REPORT TYPE } \\
\text { N/A }\end{array}$ & $\begin{array}{l}\text { 3. DATES COVERED } \\
\text { - }\end{array}$ \\
\hline \multirow{3}{*}{\multicolumn{2}{|c|}{$\begin{array}{l}\text { 4. TITLE AND SUBTITLE } \\
\text { Effects of Full Order Geopotential Hessian on Precision Orbit } \\
\text { Determination of Geodetic Satellites }\end{array}$}} & 5a. CONTRACT NUMBER \\
\hline & & 5b. GRANT NUMBER \\
\hline & & 5c. PROGRAM ELEMENT NUMBER \\
\hline \multirow{3}{*}{\multicolumn{2}{|c|}{$\begin{array}{l}\text { 6. AUTHOR }(\mathrm{S}) \\
\text { John G. Warner Krysta Lemm }\end{array}$}} & 5d. PROJECT NUMBER \\
\hline & & 5e. TASK NUMBER \\
\hline & & 5f. WORK UNIT NUMBER \\
\hline \multicolumn{2}{|c|}{$\begin{array}{l}\text { 7. PERFORMING ORGANIZATION NAME(S) AND ADDRESS(ES) } \\
\text { Naval Research Laboratory }\end{array}$} & $\begin{array}{l}\text { 8. PERFORMING ORGANIZATION } \\
\text { REPORT NUMBER }\end{array}$ \\
\hline \multirow{2}{*}{\multicolumn{2}{|c|}{ 9. SPONSORING/MONITORING AGENCY NAME(S) AND ADDRESS(ES) }} & 10. SPONSOR/MONITOR'S ACRONYM(S) \\
\hline & & $\begin{array}{l}\text { 11. SPONSOR/MONITOR'S REPORT } \\
\text { NUMBER(S) }\end{array}$ \\
\hline
\end{tabular}

12. DISTRIBUTION/AVAILABILITY STATEMENT

Approved for public release, distribution unlimited

13. SUPPLEMENTARY NOTES

This report was prepared for the AIAA 2014 SPACE Conference., The original document contains color images.

14. ABSTRACT

Successful satellite operations often depend on the ability to precisely determine the satellite's position throughout it's operational lifetime. Often, simplified models are used to reduce the computational complexity when producing an orbit determination solution. One example is the use of lower order gravitational terms when computing the state transition matrix. The US Naval Research Laboratory's Orbit Covariance Estimation and Analysis software is used to characterize the affects of using the full order geopotential terms when calculating the state transition matrix. Orbit solutions are calculated for several geodetic satellite using satellite laser ranging data. The RMS of the error residual and number of iterations needed for convergence are used as metric to evaluate the difference of using the full order geopotential to calculate the state transition matrix. It is found that typically the RMS error is the same when the higher order gravitational terms are used; however, the number of iterations needed for convergence are often lower. Additionally, the difference in the orbit solution between both methods is examined. Surprisingly, these orbit solutions may differ by up to several meters. This indicates that the higher order gravitational terms should always be considered when calculating the state transition matrix. Because fewer iterations are often needed, this can result in an overall decrease in computational time required.

15. SUBJECT TERMS

Orbit determination, Astrodynamics, geopotential, geodesy

\begin{tabular}{|c|c|c|}
\hline 16. SECURITY CLASSIFICATION OF: \\
\hline $\begin{array}{c}\text { a. REPORT } \\
\text { unclassified }\end{array}$ & $\begin{array}{c}\text { b. ABSTRACT } \\
\text { unclassified }\end{array}$ & $\begin{array}{c}\text { c. THIS PAGE } \\
\text { unclassified }\end{array}$
\end{tabular}

17. LIMITATION OF
ABSTRACT
UU

18. NUMBER OF PAGES 12 19a. NAME OF RESPONSIBLE PERSON 
Standard Form 298 (Rev. 8-98) Prescribed by ANSI Std Z39-18 
The RMS of the residual error for a 30 day fit span as well as the number of iterations required for convergence will be used as metrics. These metrics will allow for the characterization of the convergence behavior as well as the general quality of the orbit solution. Further, the resulting solution ephemerides are compared. This comparison allows for the characterization of the differences in the orbit solution.

\section{OCEAN Orbit Determination Software}

The Orbit Covariance Estimation and Analysis (OCEAN) software was developed by the Naval Center for Space Technology at the Naval Research Laboratory (NRL) to not only provide a tool to perform astrodynamics related research, but to also perform precision orbit determination to support satellite operations.

Early history of OCEAN is given in Reference 1. References 2, 3 and 4 discuss further developments of OCEAN. More recently, OCEAN has been used to calculate orbits to support operations for the NRL UPPERSTAGE and TACSAT-4 satellites.

OCEAN is a highly configurable, database driven software tool that may be used to calculate precision orbit solutions for a range of satellite missions. OCEAN allows users to simulate data, propagate a spacecraft state, or solve for an orbit using a Kalman Filter-Smoother (KFS) or Weighted Least Squares Orbit Determination (WLS-OD) process.

\section{Orbit Determination Methodology and Implementation}

Precision Orbit Determination (POD) is often accomplished by using an Extended Kalman Filter (EKF) or a batch Weighted Least Squares (WLS) process. ${ }^{5}$ In either process, the satellite dynamics are linearized around an a priori solution. For both the EKF and WLS process, the covariance matrix is propagated through time using the state transition matrix. Typically, the state transition matrix is calculated by numerically integrating the dynamics matrix ${ }^{6} .{ }^{7}$

Solving for the state transition matrix is equivalent to solving initial-value problems beginning with:

$$
\dot{\mathbf{x}}_{i}(t)=\mathbf{A} \mathbf{x}_{i}(t), \quad t \in \Re, \quad \mathbf{x}_{i}\left(t_{0}\right)=\mathbf{e}_{i} \in \Re^{n}
$$

Due to this system being time-variant, the state transition matrix is defined as the following for all $t, t_{0}$ $\in \Re$ :

$$
\mathbf{\Phi}\left(t, t_{0}\right)=e^{\mathbf{A}\left(t-t_{0}\right)}=\sum_{k=0}^{\infty} \frac{\mathbf{A}^{k}\left(t-t_{0}\right)^{k}}{k !}
$$

Where $t_{0}$ and $t$ are the start and end time respectively, $\boldsymbol{\Phi}$ is the state transition matrix, and $\mathbf{A}$ is the time varying dynamics matrix in the general, linearized state space model. For the derivation of this state transition matrix and related results, see Reference 8.

The following equations for the state transition matrix are true.

$$
\dot{\mathbf{\Phi}}=\mathbf{A} \boldsymbol{\Phi}, \quad \boldsymbol{\Phi}\left(t_{0}\right)=\mathbf{I}
$$

This allows the state transition matrix to be numerically integrated using the dynamics matrix.

In the case of the EKF, the covariance is used to determine the Kalman gain, which is used to calculate the state update. In the case of the WLS process, the covariance at each measurement is propagated to the estimation epoch where it is then used in the normal equations to calculate the state estimate. The covariance is propagated through time using the following equation.

$$
\mathbf{P}_{k}=\boldsymbol{\Phi}\left(t_{k}, t_{k-1}\right) \mathbf{P}_{k-1} \boldsymbol{\Phi}^{T}\left(t_{k}, t_{k-1}\right)
$$

Here, $\Phi_{k}$ is the covariance at time step $k$ and $\Phi\left(t_{k}, t_{k-1}\right)$ is the state transition matrix from time step $k-1$ to time step $k$.

By using higher fidelity modeling the dynamics matrix, a more precise state transition matrix is calculated and therefore a more precise covariance is calculated throughout time. Reference 9 gives more detail on the mathematics of an EKF and WLS process as applied to orbit determination.

To demonstrate how the dynamics matrix relates to the covariance matrix, first consider the equations of motion for a satellite acted on by only gravitational forces. 


$$
\ddot{\mathbf{r}}=\mathbf{g}=\nabla V
$$

where $V$ is the geopotential. ${ }^{10}$

For a state $\mathbf{x}=(x, y, z, \dot{x}, \dot{y}, \dot{z})$, the dynamics matrix $\mathbf{A}$ is defined in Reference 11 and summarized in Reference 10.

$$
\begin{aligned}
\dot{\mathbf{x}} & =\mathbf{A} \mathbf{x} \\
\mathbf{A} & =\left(\begin{array}{cccccc}
0 & 0 & 0 & 1 & 0 & 0 \\
0 & 0 & 0 & 0 & 1 & 0 \\
0 & 0 & 0 & 0 & 0 & 1 \\
\Gamma_{x, x} & \Gamma_{y, x} & \Gamma_{z, x} & 0 & 0 & 0 \\
\Gamma_{x, y} & \Gamma_{y, y} & \Gamma_{z, y} & 0 & 0 & 0 \\
\Gamma_{x, z} & \Gamma_{y, z} & \Gamma_{z, z} & 0 & 0 & 0
\end{array}\right)
\end{aligned}
$$

where

$$
\Gamma_{x, x}=\frac{\partial^{2} V}{\partial x^{2}}, \quad \Gamma_{y, y}=\frac{\partial^{2} V}{\partial y^{2}}, \quad \Gamma_{z, z}=\frac{\partial^{2} V}{\partial z^{2}}, \ldots
$$

$\Gamma$ represents the components of the gravity gradient matrix, or Hessian of the geopotential, in the rectangular inertial coordinates. ${ }^{10}$

As a simplification, the Hessian of the geopotential is often calculated by considering lower order gravity terms (just $J_{2}$ ) as demonstrated in Reference 12. However, Reference 10 derives the expressions for the full order Hessian of the geopotential in terms of the solution to Laplace's equation. This allows the state transition matrix to be integrated using arbitrary order geopotential model. Both the method from Reference 12 and Reference 10 are implemented within OCEAN and will be used to demonstrate impact of these methods on the WLS orbit determination process.

Once data is processed computationally, it will be compared to SLR results provided by the International Laser Ranging Service (ILRS). ILRS provides this data for several retro-reflector fitted spacecraft in order to support geodetic research activities. Some of the supported scientific and operational data products include Earth Orientation Parameters, Fundamental Physical Constants, Monitoring Earth Rotation and Polar Motion, and Time-Varying Geocenter Coordinates. For a full list of capabilities refer to Reference 13.

Orbit solutions are calculated using the OCEAN WLS-OD process for 30 days of data. This long duration orbit fit demonstrates how well the long term satellite dynamics can be modeled. The RMS residual error is used as a metric to demonstrate how well the data are fit. Further, the number of iterations needed for convergence are tracked. This allows the effects on the solution convergence for each method to be tracked. Last, orbit ephemeris solutions are compared to determine the effects of both methods on the solution.

The OCEAN WLS-OD capability employs extensive spacecraft, measurement, and force modeling to estimate the desired spacecraft state and parameters. Force models include solid Earth tides; pole tides; lunar and solar third body gravitational effects; indirect lunar oblateness; general relativistic effects; atmospheric variability; drag; and solar radiation pressure. OCEAN WLS-OD accounts for various time systems, including TAI, UTC, and GPS time. The WLS-OD functionality also uses Earth Orientation Parameter (EOP) models from the International Earth Rotation and Reference Systems Service (IERS), which account for precession and nutation, Earth rotation, and polar motion. OCEAN implements the majority of the conventions recommended in IERS Conventions 2010. ${ }^{14}$ For this application, a ninth order multi-step predictor-corrector algorithm is used to perform the integration of the state variables and state transition matrix. OCEAN will be used with SLR data to calculate precision orbits for several geodetic satellites, including, LAGEOS-1, LAGEOS-2, STARLETTE and STELLA. These satellites are well suited for orbit determination analysis as their orbit was designed to be sensitive only to the well characterized gravity forces and minimally sensitive to atmospheric drag and radiation pressure forces. Details for each satellite are as follows.

\section{A. LAGEOS-1}

The LAGEOS-1 satellite was launched in 1976 by the National Aeronautics and Space Administration (NASA) as part of the Earth and ocean dynamics application program. It was designed to provide a long- 
lasting laser target in a well defined orbit. This satellite enabled researchers to study a range of geophysical phenomena with improved accuracy, including the Earth's geopotential.

The satellite's low ballistic coefficient combined with its spherical shape minimize the orbital uncertainty due to drag and solar radiation forces.

The nominal orbital elements are given in Table 1.

Table 1. Nominal Orbital Elements for LAGEOS-1

\begin{tabular}{|l|c|}
\hline Element & Nominal Value \\
\hline \hline Semi-major Axis & $12,240 \mathrm{~km}$ \\
Eccentricity & 0.0045 \\
Inclination & $109.84^{\circ}$ \\
\hline
\end{tabular}

\section{B. LAGEOS-2}

The LAGEOS-2 satellite was launched in 1992 by NASA and the Agenzia Spaziale Italiana (ASI). The LAGEOS-2 satellite is an almost identical design to the LAGEOS-1 satellite; however, its orbit was selected to provide increased coverage over seismically active areas such as the Mediterranean and California.

The nominal orbital elements for LAGEOS-2 are given in Table 2.

Table 2. Nominal Orbital Elements for LAGEOS-2

\begin{tabular}{|l|c|}
\hline Element & Nominal Value \\
\hline \hline Semi-major Axis & $11,998 \mathrm{~km}$ \\
Eccentricity & 0.0135 \\
Inclination & $52.64^{\circ}$ \\
\hline
\end{tabular}

\section{STARLETTE}

The STARLETTE satellite was launched in 1975 by Centre Nationale d'Etudes Saptiales (CNES). The spacecraft was designed to improve the geopotential model and to study solid Earth tides, ocean tides, and polar motion. ${ }^{15}$ STARLETTE was also the first spacecraft to be entirely covered by laser corner reflectors which allow passive SLR observation capabilities. ${ }^{16}$ The STARLETTE orbit is highly sensitive to zonal variations in the gravity field.

The nominal orbital elements for STARLETTE are given in Table 3.

Table 3. Nominal Orbital Elements for STARLETTE

\begin{tabular}{|l|c|}
\hline Element & Nominal Value \\
\hline \hline Semi-major Axis & $7,190 \mathrm{~km}$ \\
Eccentricity & 0.0206 \\
Inclination & $49.83^{\circ}$ \\
\hline
\end{tabular}

\section{STELLA}

The STELLA satellite was launched in 1993 by CNES, and is virtually identical to the STARLETTE satellite. As with the LAGEOS satellites, both STELLA and STARLETTE are spherically-shaped spacecraft with low ballistic coefficients to minimize orbital uncertainty caused by drag and solar radiation pressure forces.

The nominal orbital elements for the STELLA satellite are given in Table 4. 
Table 4. Nominal Orbital Elements for STELLA

\begin{tabular}{|l|c|}
\hline Element & Nominal Value \\
\hline \hline Semi-major Axis & $7,178 \mathrm{~km}$ \\
Eccentricity & 0.0206 \\
Inclination & $98.6^{\circ}$ \\
\hline
\end{tabular}

\section{Testing Results}

The International Laser Ranging Service (ILRS) provides global satellite laser ranging data in order to support geodetic research activities. Reference 13 contains further information in regards to the services and capabilities provided. The ILRS catalogs SLR data to a number of geodetic satellites. These satellites are typically design to facilitate the study of Earth's gravity and contain laser retro-reflectors. This allows highly precise laser ranging measurements to made which are well suited for performing precision orbit determination.

SLR data from the LAGEOS-1, LAGEOS-2, STARLETTE and STELLA satellites will be used to evaluate the impact of using both the state transition matrix calculated using only the $J_{2}$ component of Earth's gravity as well as the state transition matrix calculated using the EGM2008 geopotential model to an arbitrary order. The RMS of the residuals as well as the number of iterations needed for convergence are shown. Additionally, differences in the calculated orbit ephemerides are presented.

\section{A. LAGEOS-1 Results}

Table 5 shows the RMS of the error residuals and number of iterations needed for convergence for the 30 day WLS-OD solution for the LAGEOS-1 satellite. As can be seen, the RMS of the error residuals are nearly identical when either the $J_{2}$ gravity partial derivatives or the full order gravity partial derivatives are used. However, the number of iterations needed for convergence is reduced by two.

Figure 1 shows the RSS of the orbit position difference between the two solutions. While the orbit solutions are not identical, they only vary on the order of one millimeter. This difference is negligible.

Table 5. Results from LAGEOS-1 30-day Orbit Solution

\begin{tabular}{|l|c|c|}
\hline Method & Error Residual RMS & Iterations \\
\hline$J_{2}$ & $50.95 \mathrm{~cm}$ & 10 \\
\hline Full Order & $50.96 \mathrm{~cm}$ & 8 \\
\hline
\end{tabular}

\section{B. LAGEOS-2 Results}

Table 6 shows the RMS of the error residuals and number of iterations needed for convergence for the 30 day WLS-OD solution for the LAGEOS-2 satellite. As can be seen, the RMS of the error residuals are similar when either the $J_{2}$ gravity partial derivatives or the full order gravity partial derivatives are used. The orbit solution when using the full order gravity partial derivatives does yield slightly lower error residuals. Similar to the results from the LAGEOS-1 orbit solution, the number of iterations needed for convergence is reduced by two when using the full order gravity partial derivatives.

Figure 2 shows the RSS of the orbit position difference between the two solutions. Here, the orbit solutions vary on the order of several centimeters. This difference is manifested primarily as an in-track orbit difference between the two satellites.

Table 6. Results from LAGEOS-2 30-day Orbit Solution

\begin{tabular}{|l|c|c|}
\hline Method & Error Residual RMS & Iterations \\
\hline$J_{2}$ & $57.05 \mathrm{~cm}$ & 11 \\
\hline Full Order & $56.98 \mathrm{~cm}$ & 9 \\
\hline
\end{tabular}




\section{STELLA Results}

Table 7 shows the RMS of the error residuals and number of iterations needed for convergence for the 30 day WLS-OD solution for the STELLA satellite. As can be seen, the RMS of the error residuals is higher by nearly 25 centimeters when using $J_{2}$ gravity partial derivatives as opposed to the full order gravity partial derivatives. Interestingly, the number of iterations needed for convergence is the same in both cases.

The RMS of the error residual is much higher here than in either LAGEOS-1 or LAGEOS-2 orbit solutions. This is due to the nature of the STELLA orbit, which was designed to be sensitive to tidal effects and temporal variations of the geopotential. These are often more difficult to model over longer time spans.

Figure 3 shows the RSS of the orbit position difference between the two solutions. Here, the difference in the orbit solutions is much more pronounced. The RSS of the position difference can vary by as much as 3.5 meters. This difference is manifested mainly as a cross-track orbit difference (which accounts for about 2 meters average difference), and to a lesser degree as an in-track orbit difference (which accounts for about 1 meter average difference).

Table 7. Results from STELLA 30-day Orbit Solution

\begin{tabular}{|l|c|c|}
\hline Method & Error Residual RMS & Iterations \\
\hline$J_{2}$ & $6.401 \mathrm{~m}$ & 8 \\
\hline Full Order & $6.149 \mathrm{~m}$ & 8 \\
\hline
\end{tabular}

\section{STARLETTE Results}

Table 8 shows the RMS of the error residuals and number of iterations needed for convergence for the 30 day WLS-OD solution for the STARLETTE satellite. As can be seen, the RMS of the error residuals is higher by nearly 14 centimeters when using $J_{2}$ gravity partial derivatives as opposed to the full order gravity partial derivatives. Here, the reduction in the number of iterations needed for convergence is the most pronounced, requiring half the number of iterations when using the full order gravity partial derivatives as opposed to the $J_{2}$ gravity partial derivatives.

Again, the RMS of the error residuals are higher than either the LAGEOS-1 or LAGEOS-2 orbit solutions. Similarly to STELLA, the STARLETTE orbit was designed to be sensitive to tidal effects and temporal variations of the geopotential. These are more difficult to model over longer time spans.

Figure 1 shows the RSS of the orbit position difference between the two solutions. Here, there is an average orbit difference of about 1 meter. This is primarily manifested as an in-track orbit difference.

Table 8. Results from STARLETTE 30-day Orbit Solution

\begin{tabular}{|l|c|c|}
\hline Method & Error Residual RMS & Iterations \\
\hline$J_{2}$ & $4.207 \mathrm{~m}$ & 12 \\
\hline Full Order & $4.071 \mathrm{~m}$ & 6 \\
\hline
\end{tabular}

\section{Conclusions}

The result show that using the full order geopotential when calculating the components of the gravity gradient matrix can significantly reduce the number of iterations needed for the WLS orbit determination process (by as much as a factor of two). While calculating the full order Hessian is more computationally intensive than calculating the $J_{2}$ gravity terms, the reduction in the number of iterations still can decrease the total execution time. This is due to the fact that execution time for the WLS-OD is almost linearly related to the number of iteration required.

Interestingly, by using the full order geopotential Hessian, the RMS of residual error can vary appreciably. This variation is much more pronounced for the STELLA and STARLETTE satellites, which are in orbits that are sensitive to higher order geopotential terms. In those cases, the solution orbits can differ significantly. Overall, these higher order gravitational terms should be used in a WLS-OD process. 
Ultimately, it is shown that by using the full order geopotential a substantial decrease in number of iterations required for convergence can be realized and a potentially better orbit solution may be reached.

Future work includes examining how many higher order gravitational terms are necessary to realize these gains and to better characterize this method's performance for satellites in different orbit regimes. More work is needed to characterize the convergence behavior of both methods. It is possible that by incorporating higher order terms in the Hessian of the geopotential that radius of convergence in the WLS-OD method is increased. 


\section{References}

${ }^{1}$ Soyka, M. T., Middour, J. W., Binning, P. W., Pickard, H., and Fein, J., "The Naval Research Laboratory's orbit/covariance estimation and analysis software- OCEAN," Astrodynamics 1997, 1997, pp. 1567-1586.

${ }^{2}$ Soyka, M. T., Middour, J. W., and Fein, J., "Simultaneous orbit determination of large satellite constellations," Spaceflight mechanics 1998, 1998, pp. 1275-1293.

${ }^{3}$ Binning, P. W., Soyka, M. T., and Middour, J. W., "Orbit determination using space to ground Differential GPS in NRL's OCEAN package," Astrodynamics 1999, 2000, pp. 421-434.

${ }^{4}$ Seago, J. H., Davis, M. A., Smith, W., Fein, J., Brown, B., Middour, J., Soyka, M., and Lydick, E., "More results of naval space surveillance system calibration using satellite laser ranging." Advances in the Astronautical Sciences, Vol. 112, 2002, pp. 1177-1196.

${ }^{5}$ Vetter, J. R., "Fifty Years of Orbit Determination," Johns Hopkins APL technical digest, Vol. 27, No. 3, 2007, pp. 239.

${ }^{6}$ Terejanu, G. A., "Extended Kalman Filter Tutorial," Nov. 212011.

${ }^{7}$ Fadali, M. S., "Least-Squares Estimation: Batch Processing," Sept. 292013.

8 "EE 580 - Linear Control Systems: VI. State Transition Matrix," Aug. 82010.

${ }^{9}$ Tapley, B. D., Schutz, B. E., and Born, G. H., Statistical Orbit Determination, Elsevier Academic Press, 2004.

${ }^{10}$ Melvin, P. J., "A Kalman filter for orbit determination with applications to GPS and stellar navigation," Spaceflight mechanics 1996, 1996, pp. 719-738.

${ }^{11}$ Battin, R. H., "A statistical optimizing navigation procedure for space flight," ARS Journal, Vol. 32, No. 11, 1962, pp. 1681-1696.

${ }^{12}$ Markley, F., "Approximate Cartesian state transition matrix," Journal of the Astronautical Sciences, Vol. 34, 1986, pp. 161-169.

${ }^{13}$ Pearlman, M.R., D. J. and Bosworth, J., "The International Laser Ranging Service," 2002.

${ }^{14}$ Petit, G. and Luzum, B., "IERS conventions (2010)," Tech. rep., DTIC Document, 2010.

${ }^{15}$ Kanner, L. and Associates, "Translation of 'Le satellite de geodesie 'Starlette', Groupe de Recherches de Geodesie Spatiale, Centre National d'Etudes Spatiales, Bretigny-sur-Orge, France, Report, 1974, 25 pp," National Aeronautics and Space Administration, Washington, D. C., July 1974.

${ }^{16}$ Kramer, H. J., Observation of the Earth and Its Environment: Survey of Missions and Sensors, Springer, 2002. 
Figure 1. LAGEOS-1 RSS Orbit Difference Between Full Order Partials Solution and J2 Partials Solution

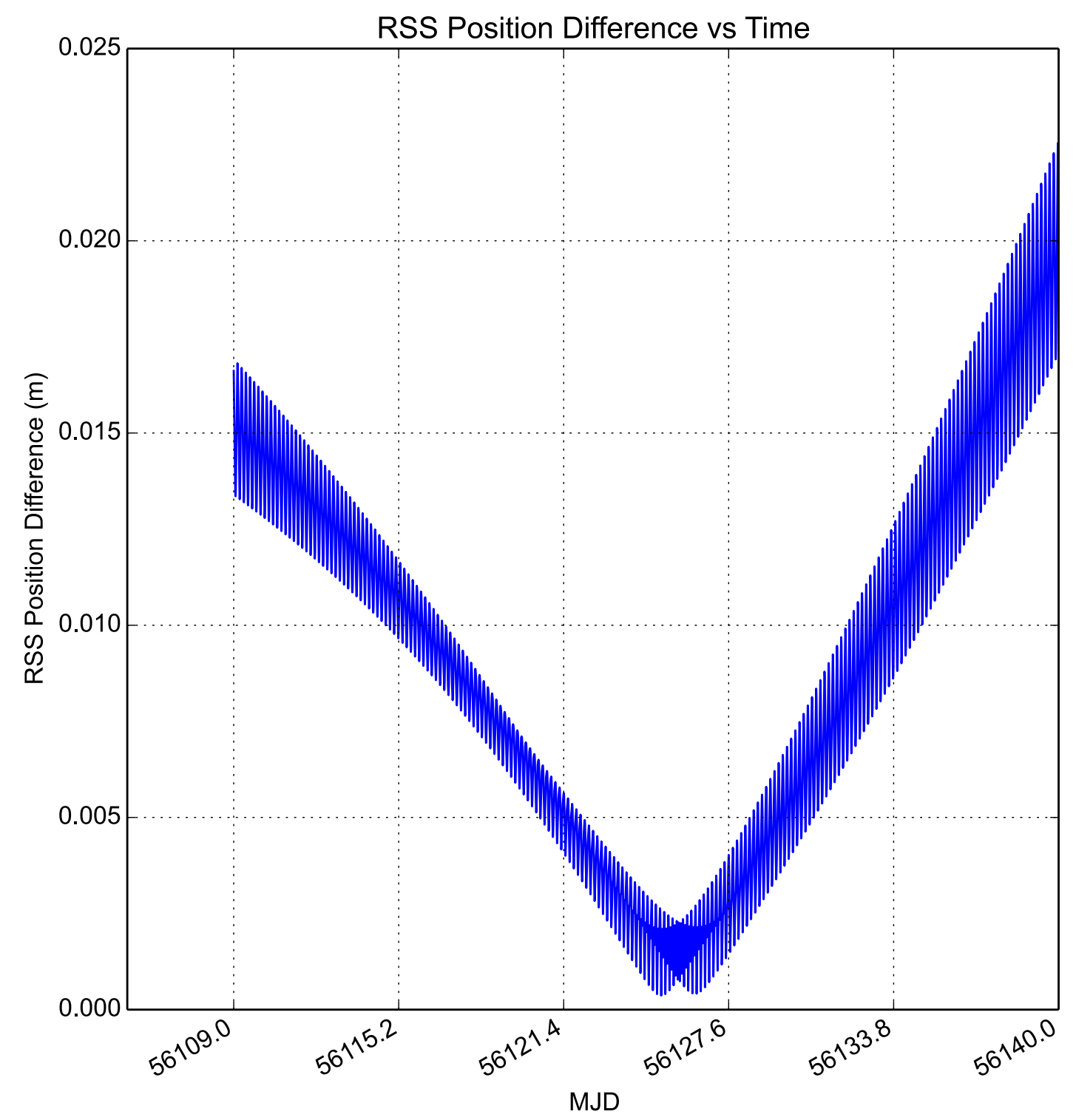


Figure 2. LAGEOS-2 RSS Orbit Difference Between Full Order Partials Solution and J2 Partials Solution

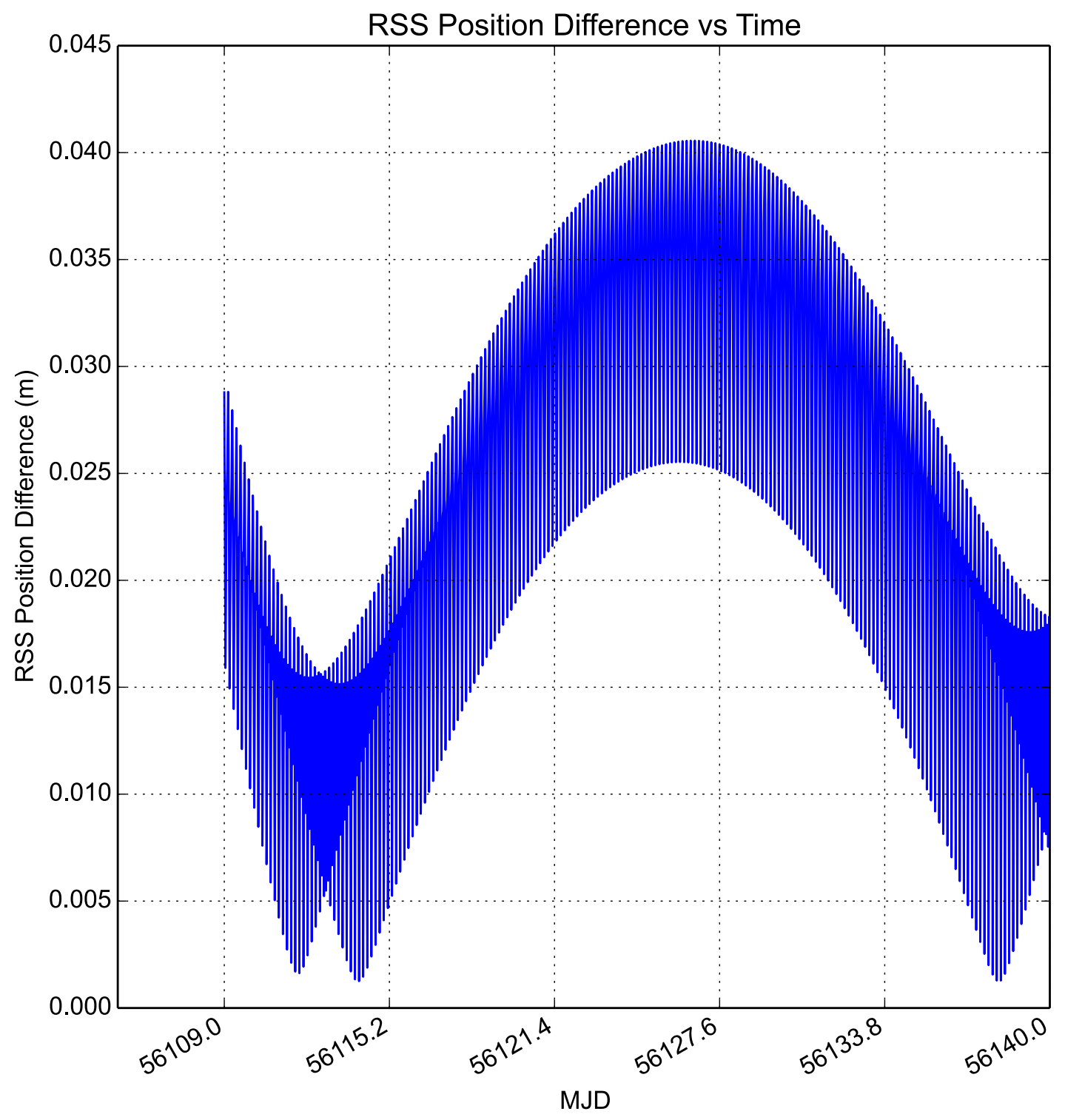


Figure 3. STELLA RSS Orbit Difference Between Full Order Partials Solution and J2 Partials Solution

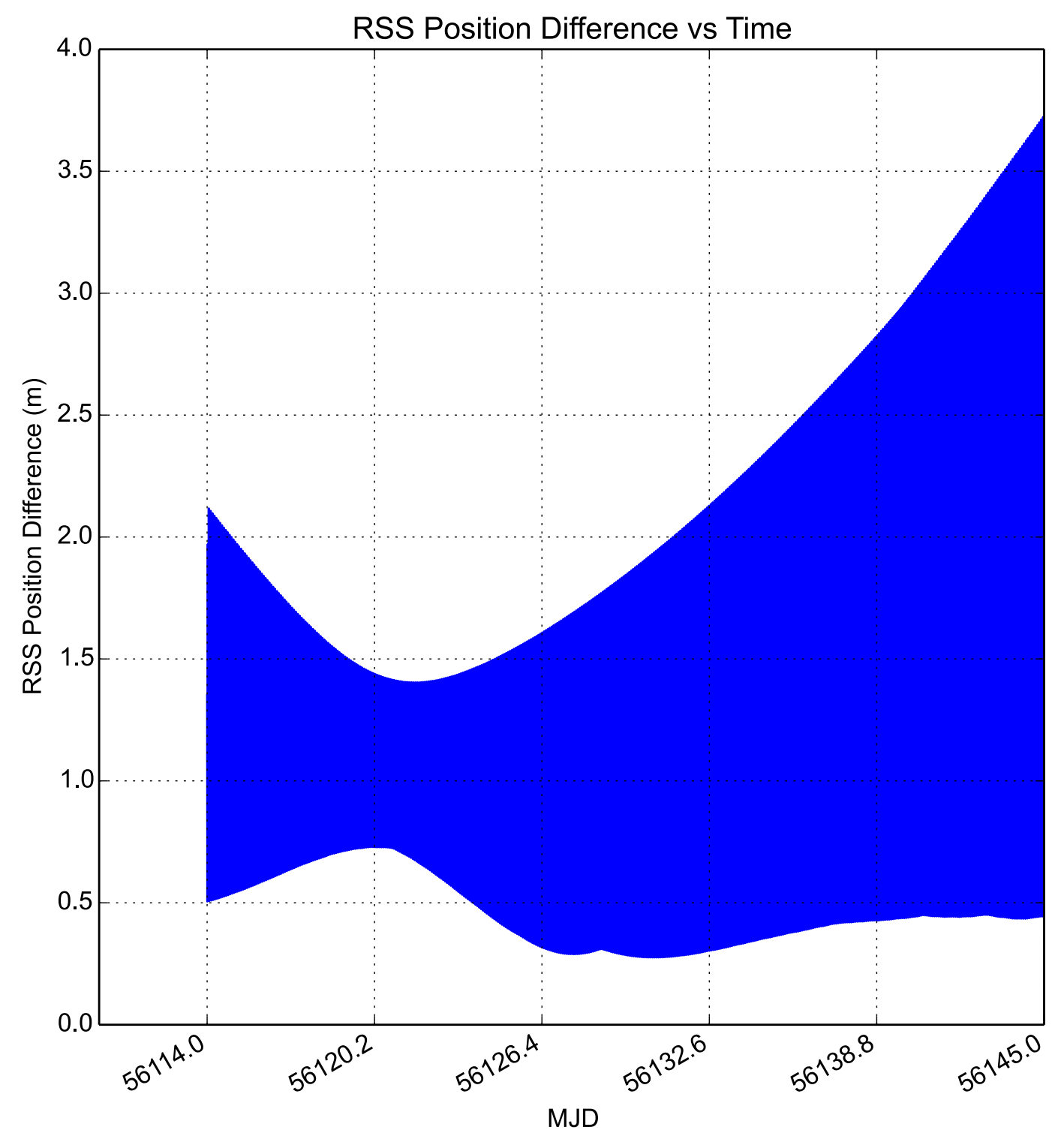


Figure 4. STARLETTE RSS Orbit Difference Between Full Order Partials Solution and J2 Partials Solution

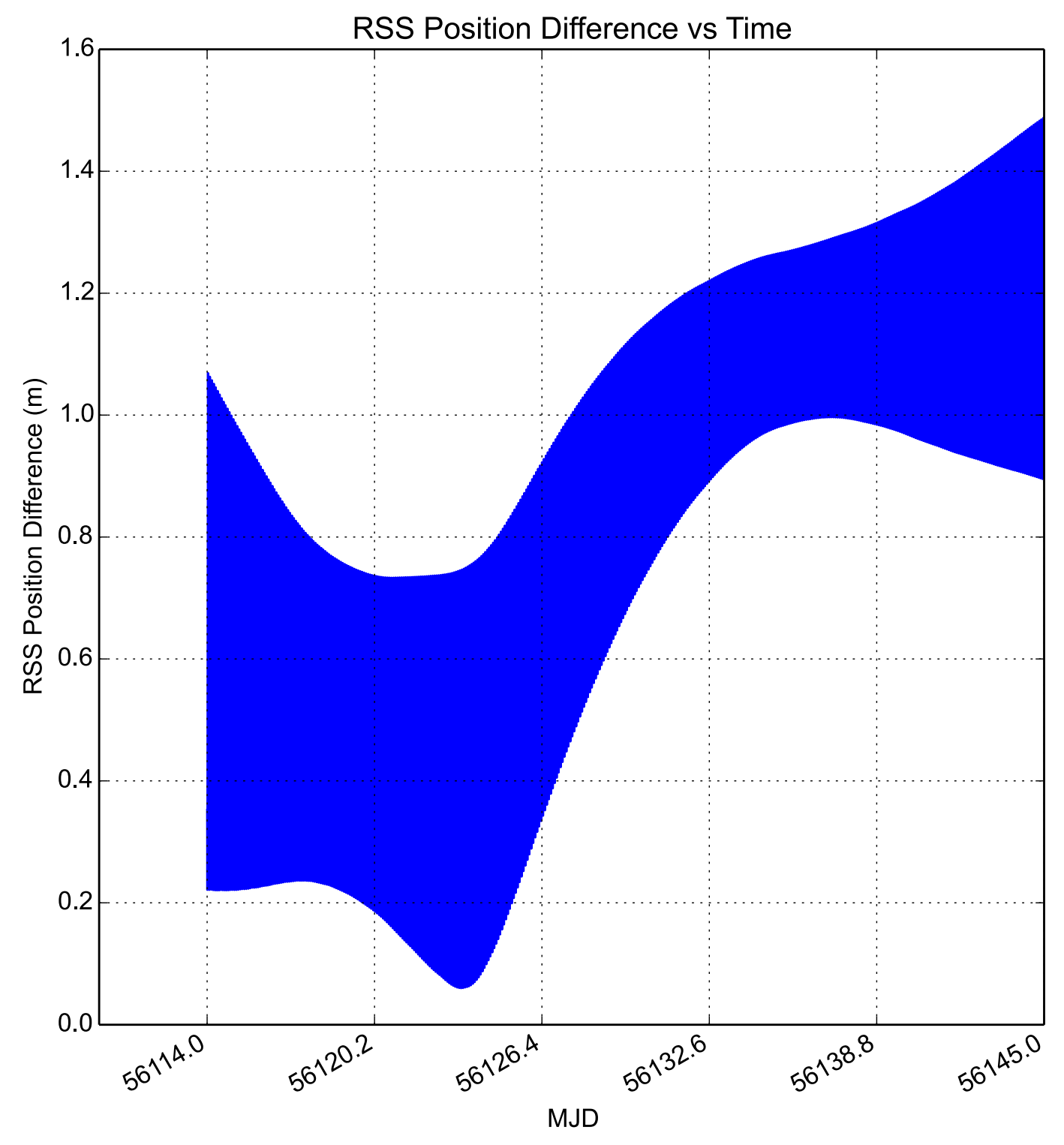

